\title{
Refueling the Magic Furnace: Kilonova 2017 Rewrites the Story of Element Origins
}

\author{
Barry Wood \\ University of Houston
}

\begin{abstract}
For more than half a century, we have understood element creation in the stars - described in Marcus Chown's colorful image as "the magic furnace." From 1958 until 2017, supernova explosions were thought to be the primary site of element creation above Iron, No. 26 on the Periodic Table. This assumption was radically overturned with the August 17, 2017, arrival of signals from a catastrophic collision of neutron stars. This paper traces the history of element-creation science leading to the striking discoveries attending "Kilonova 2017" that now call for a rewriting of the nucleosynthesis chapter of Big History.
\end{abstract}

Correspondence | Barry Wood, barrywood1940@yahoo.com

Citation | Wood, B. (2018) Refueling the Magic Furnace: Kilonova 2017 Rewrites the Story of Element Origins. Journal of Big History, II(3); 1 - 15.

DOI | http://dx.doi.org/10.22339/jbh.v2i3.2300

\section{Introduction}

Pangea had recently broken up ... North America and Europe were slowly drifting apart

... the Himalayas had not yet appeared . . . tropical jungles harbored enormous predatory dinosaurs that would roam the earth for another 65 million years ... mammals the size of housecats kept to the shadows . . . squirrel-sized primates cowered in caves ...

Then ...

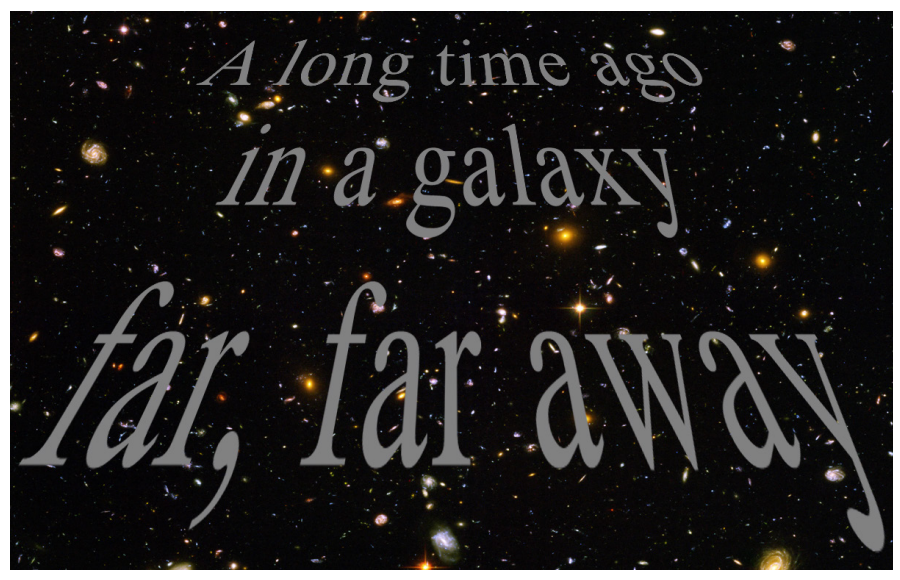

an explosion of monumental proportions

Vibrations radiated out in all directions, traveling at 300,000 kilometers $(186,000$ miles) per second. One hundred and thirty million years later, on August 17, 2017, a unique signal reached Earth, detected by three recently constructed gravitational wave (GW) detectors in Italy, Louisiana, and Washington State. ${ }^{1}$
Beginning at $24 \mathrm{Hertz}$, it gradually rose over 100 seconds to several hundred Hertz - indicating the inspiraling of two massive bodies about to detonate in a cataclysmic collision. Following 3,000 cycles, the GW signal came to an abrupt end at 12:41:04.4 UTC (Coordinated Universal Time). Almost immediately (1.74 seconds later) a two-second gamma-ray burst was detected by the orbiting Fermi Gamma-ray Space Telescope (FGST) launched in 2008. Computers went to work, triangulating from signals milliseconds apart to identify a multi-moon sized region in the sky. Within a minute FGST had identified a range and region in the sky, narrowing the search to 100 galaxies. Alerts went out around the world. Every active astronomy team tuned in on the event, receiving signals that would eventually be combined into a minute-by-minute record of a neutron star collision - designated by its gravity waves and date of observance as GW170817. By year's end, this had become the most thoroughly documented astronomical event in history.

The simultaneous arrival of gravity waves and gamma rays from a source 130 million light years away confirmed the velocity of gravity waves as equal to the speed of light. But gravity waves and

I would like to thank the anonymous reviewers, both physicists, who provided corrections, valuable suggestions, and additional references to strengthen the article. 
gamma rays are invisible, while humans are attuned to visible light, images, photographs constructed from the electromagnetic spectrum. Following a systematic search through the " $90 \%$ confidence region," aided by comparisons with archival records, four Carnegie astronomers using the Swope Campanas Observatory in Chile located the light signal in NGC 4993, a distant galaxy known since John Herschel discovered it in 1789. The source, identified 10.9 hours after the event, was officially designated Swope Supernova Survey 2017a (SSS 2017a). ${ }^{2}$ The account of this search is itself a dramatic story. The area of interest was viewable only from the southern hemisphere, thus eliminating northern observatories from direct viewing. Obscured by sunlight, observations were delayed for 10.9 hours until the southern hemisphere rotated into night - a delay that heightened anticipation of an optical record unique in astronomical history. The electromagnetic record ranged from visible light to infrared, allowing for precise spectral analysis. Five more teams provided primary observational platforms; seventy observatories around the world tuned in, with thousands of astronomers following this event, recognizing that this conjunction of gravity waves, gamma rays, and visible light had just ushered in a new era of "multi-messenger" astronomy. ${ }^{3}$

\section{A Unique Moment in Science}

As we look over the past two or three centuries, a handful of significant events have changed our understanding of the universe and provided a new beginning in the unfolding narrative of scientific discovery. Some reside in publications whose full significance has unfolded over decades, like Darwin's Origin of Species (1859) or Einstein's Special Theory of Relativity (1905). Others consist of a landmark discovery: James Hutton's 1794 recognition of "deep time" visible in the geological unconformity at Siccar Point; Edwin Hubble's 1929 observations of distant galaxies retreating according to a systematic constant. More recently, teams of scientists seeking precise confirmation for signals from deep space, detected by the Holmdel Horn Antenna in 1965, discovered it when the Cosmic Microwave Background (CMB), was finally photographed by the Cosmic Background Explorer (COBE). The image made news around the world in 1990.

Astronomers have long awaited direct evidence of gravity waves proposed by Einstein's work. Indirect evidence of gravitational waves from orbital-period variations in a pulsar binary date to 1970 and won a Nobel Prize in physics in 1993. But direct evidence was long delayed. The physics of theoretical cosmic events led to predictions of neutron-star collisions decades ago, though as $\mathrm{Li}-\mathrm{Xin} \mathrm{Li}$ and Bohdan Paczynski (1988) recognized, a mathematical model "can provide only an order-of-magnitude estimate of peak luminosity and the timescale of the transient event that is likely to follow a violent merger of two neutron stars." ${ }^{\prime 4}$ It is notable that $\mathrm{Li}$ and Paczinski did not mention gravity waves or gamma rays in 1988, focusing instead on light —our primary window into the universe for centuries - an indication of how the dominant technology of light-gathering telescopes limited the kind of evidence available for analysis.

Detecting gravity waves required inventing a device capable of measuring space-time perturbations smaller than the width of an atomic nucleus. The whole forty-year story from idea to invention is told by Jenna Levin in Black Hole Blues and Other Songs from Outer Space (2017), along with the way this enormous project was intertwined with the lives and ambitions of its inventors. The instrument was imagined and designed in the 1970s, but development was halting, at times stopped, though eventually completed with massive funding; in the end, it was the most highly funded project in the history of astronomy. Two detectors eventually came online in Washington State and Louisiana, with a third in Italy, and gravity waves were detected four times from 2015 until early 2017. These were thought to be collisions of black holes or perhaps the swallowing of a massive star by a black hole. For the success of their decades-long project, a Nobel Prize was awarded to three long-term principals, 
the limit for prize sharing, though many contributed to its design, and development engaged more than 1000 researchers. Deciding who should receive the award veered toward political conflict: at least four were deserving of the prize, but the decision was simplified by the March 2017 death of physicist co-founder Ronald Dever at the age of 85. Rainer Weiss, Kip Thorpe, and Barry Barish thus shared the Nobel Prize in physics, awarded in October 2017. Weiss, who set forth the key design for the detector in a paper in 1972, generously commented, "we're symbols for the much bigger group of people who made it happen," and Barish added, "I have somewhat ambivalent feelings about the recognition of individuals when so much of this was a team effort." 5 The Prize, announced early in October 2017, provided a capstone for one of the most ambitious projects in physics. By this time Kilonova 2017 had demonstrated gravitational waves leading to merger. once again; its dramatic announcement would soon follow.

The discovery and coordination of data was kept under wraps for three months until its official announcement in mid-November 2017, at which point a term for a neutron-star collision, "kilonova," proposed by B. D. Metzger et al (2012), suddenly achieved common currency in the vocabulary of science. ${ }^{6}$ Nothing has approached the flood of scientific publication attending this announcement, including advanced papers and more than 100 article preprints. Press releases appeared from dozens of news agencies and universities. Six letters appeared in the leading British publication, Nature; eight in the flagship

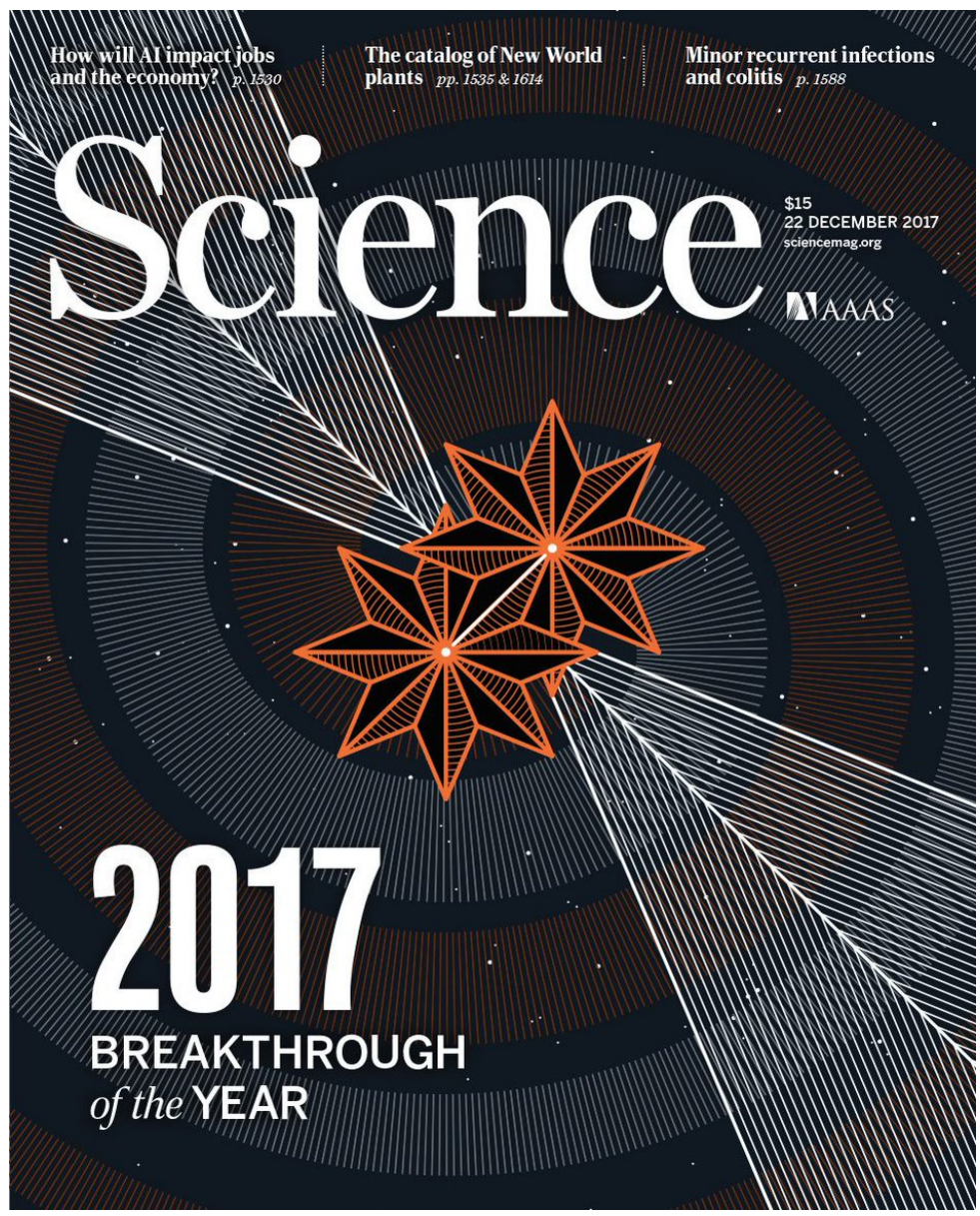

Cover of Science Magazine, December 22, 2017, depicting the rotating laser-like pulsar beams and inspiraling neutron stars AAAS weekly, Science, with 32 in a special issue of The Astronomical Journal Letters; the lead article (149 pages) coordinating observations from 70 observatories was coauthored by 4,000 astronomers from 900 institutions. $^{7} \quad$ The 22 December 2017 issue of Science hailed this "cosmic convergence" of neutron stars as the breakthrough story of the year, marking the announcement with six more research papers. ${ }^{8}$

\section{The Significance for Big History}

One of the biggest problems for teachers of Big History is deciding what is important enough for incorporation into our cosmic narratives. Even a fraction of what we know may be too much. Beginning with astronomy, our territory ranges through physics, chemistry, biology, ecology, anthropology, sociology, psychology, and a welter of historical and cultural strands most of us are still sifting for their significance. Our goal is to explore and construct a continuous narrative that links Cosmos, Earth, Life, Humanity, and Culture, but the separate silos of our disciplines lure us toward our specialties. Astronomers 
feel comfortable up to the creation of Earth and the Hadean era; geologists focus on of rocks, mountains, and plate tectonics; historians find significance in the Holocene-from the Agricultural Revolution to the Internet. None of us are quite sure how to weight the Anthropocene chapter, which is so central for the present human situation that it threatens to overwhelm the preceding 13.8-billion-year narrative.

Here we are easily influenced by the enthusiasm of specialists who observe these events. A neutron star merger has an exotic appeal for astronomers because it is so rare, but just how do infinitesimal gravity waves attending relatively rare events in faraway galaxies concern us? Or, if they occur closer to home somewhere in our own galaxy, should they be part of the big story? Pretelescopic sightings of nearby supernovae $(1054,1572,1604)$ indicate these as sporadic, sometimes centuries apart, but more thorough sky surveys now suggest one or two in our galaxy every century. In contrast, a Milky Way neutron-star collision is estimated to occur once every 10,000 years. We are therefore far more likely to find them elsewhere. But gravity waves seem distant, especially when we learn that the August 17, 2017, collision produce vibrations in our own space-time framework one thousandth the width of a proton. Our technological ability to measure perturbations this fine seems more significant than the perturbations themselves!

But, the optical data attending this event is another story. In the hours following the collision, a shower of heavy elements appeared. Most reports havementioned gold and platinum-elements that hold particular significance for humans - but these are the tip of the iceberg or, in a more relevant metaphor, the top drawer of the mineral cabinet. The total of heavy elements observed added up to an estimated 16,000 Earth masses. We now recognize that neutron star collisions produce more than half of the sixty-six elements above Iron, number 26 on the Periodic Table. This moves the significance of this event to the mainstream of the Big History story. Element creation is a fundamental chapter that falls between the emergence of Time, Space, Matter, and Energy when everything started up and the later combining of elements on a wellplaced planet to form chemical molecules, and life. Thus positioned, the creation of the elements is an intermediate story of monumental importance. But with the magnificent fireworks of Kilonova 2017, we now recognize that this central chapter has long been incomplete, or regarded as complete when in fact the final episode was understood imperfectly. For this, we need to look at the story of element fusion in the stars as it unfolded over the previous eighty years.

\section{The Fusion of Hydrogen to Iron in the Stars}

The creation of the elements is a lynchpin chapter in Big History. It presents a sequence of material creativity before the much more complex innovations at the molecular, cellular, and organic levels. It also provides an arena for exploring a primary idea - the astonishing and unpredictable properties that appear as particles are added; for instance, as we move from element 78 to 79 to 80 (platinum to gold to mercury), from brilliant shiny minerals to a dull and viscous liquid poisonous to life. There is hardly any better illustration of the unifying theme of emergence. Yet the story is hardly known. My big history course, Cosmic Narratives, attracts students from roughly 25 majors, including the major sciences. On questioning chemistry majors, I am always surprised that this story is not routinely covered in their classes. The reason is simple; our institutionalized silo separation of knowledge defines the creation of elements as irrelevant. This is well illustrated in a standard undergraduate chemistry text that includes a four-page section on nuclear reactors and fusion in the laboratory from "bombardment using particle accelerators," noting the role of fusion in the hydrogen bomb. The origin of the elements is treated - tentatively and anthropically_in a single sentence: "It is now believed that the energy of stars, including our sun, where extremely high temperatures exist, derives from nuclear fusion." This astonishing reduction of stellar nucleosynthesis in a chemistry 
text of 1030 pages shows professional chemists overlooking the multidisciplinary connections of their subject, disinterest in relating creative events at cosmic scale to human life, and a focus primarily on technological applications.

The fact is the story of element creation in the stars, by itself, constitutes a liberal education in the midst of astronomy, physics, and chemistry, a point where the structure of narrative from the humanities provides an organizing principle for the sciences. ${ }^{10}$ We are, after all, made of elemental materials forged billions of years ago in the stars. In this light, the contribution of Kilonova 2017 enters the picture. We need to review the story, noting the contribution of this singular event to rewriting this crucial chapter of Big History.

In an early myth, the stars were lights shining

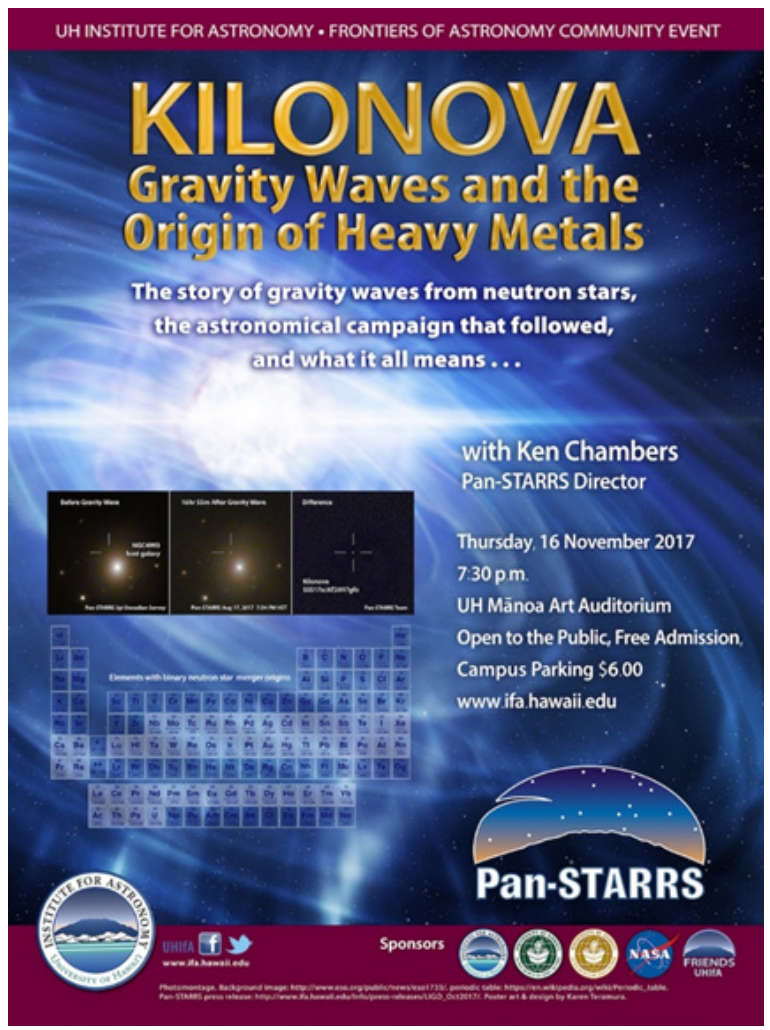

Poster for the astronomical community event sponsored by the Panoramic Survey Telescope and Rapid Response System (Pan-STARRS) at the University of Hawaii on the evening before the Kilonova 2017 announcement. Note the elements (highlighted) created by the merger. through tiny holes in the celestial sphere. An ancient cosmic narrative has the sun-god Helios driving a fiery chariot across the sky; the Egyptians named him $\mathrm{Ra}$, the Hindus Surya, the Romans Sol. The idea of the Sun's warmth as simple combustion persisted until the eighteenth century when the extended age of the Earth suggested by geologists made clear that a Sun consuming fuel like wood or coal would have long ago burned out. In the early twentieth century, radioactivity was considered, but this was associated with heavy elements like uranium and thorium whose abundance was insufficient to fuel the Sun for millions of years. A conceptual breakthrough came with Sir Arthur Eddington's classic study, The Internal Constitution of the Stars (1926) where he suggested that the source of the Sun's heat was the "annihilation of matter," an insight derived from Einstein's equivalency of matter and energy, though Eddington had no idea how the conversion might occur.

Clarification came over the next three decades as a new generation of physicists worked out the principles of nuclear fusion. The first attempt came with a seminar paper by Fred Hoyle, "The Synthesis of the Elements from Hydrogen," presented at the Royal Astronomical Society meeting on November 8, 1946. ${ }^{11}$ Hoyle's theory that synthesis occurred in stars was indeed a conceptual breakthrough; as Margaret Burbidge, who heard the paper, remarks, this was "a time when the current theory was that the elements were created primordially by the coagulation of neutrons just after the birth of the Universe."12 Hoyle was persuasive in presenting his new view, asserting that "our first object of finding a place in the universe where the elements may be synthesized has been achieved."13 Paralleling the development of particle accelerators, ${ }^{14}$ a generation of young physicists began a theoretical exploration of nuclear fusion, the idea of bombarding atomic nuclei with particles and working out how difficult barriers might be overcome. It was known that the electron shell preserves considerable space inside the atom that presents front-line stopping power; in addition, the enormous repellent force of 
positively charged protons in the atomic nucleus presents withering firepower against anything but a direct high-velocity frontal attack. Overcoming these, it was realized, would require impacts of electrically neutral neutrons and protons at enormous velocities attainable only with temperatures in the order of millions of degrees. Theory based on well-developed laws of physics held that a dead on collision with an atomic nucleus was more likely for a neutron while very difficult for a proton, but construction of a more complex nucleus depended on the addition of both protons and neutrons. Precise pathways would take much exploration.

A conceptual breakthrough had come with Harold Urey's 1932 discovery of a rare and heavy isotope of hydrogen consisting of one proton and one neutron which he called "deuterium," conveniently designated as $\left({ }^{2} \mathrm{D}\right) .{ }^{15}$ Building on this, Charles Critchfield, who had studied under the Russian astronomer George Gamow, recognized that deuterium was a possible intermediate step in what eventually was called the proton-proton process, each step of which would result in some matter converted to energy. ${ }^{16}$ The process is easy to follow:

${ }^{1} \mathrm{H}+{ }^{1} \mathrm{H}$ yields ${ }^{2} \mathrm{D}+$ energy: a neutrino and a positron $(\mathrm{e}+)$ combine with a regular electron

(e-) and are radiated as a gamma ray

${ }^{2} \mathrm{D}+{ }^{1} \mathrm{H}$ yields ${ }^{3} \mathrm{H}+$ energy: a gamma ray

The end product, ${ }^{3} \mathrm{H}$, is incomplete helium missing one neutron, thus requiring another step;

${ }^{3} \mathrm{H}+{ }^{3} \mathrm{H}$ yields ${ }^{4} \mathrm{H}$ (Helium) $+{ }^{1} \mathrm{H}+{ }^{1} \mathrm{H}$

(which can begin the process again)

While Critchfield developed the proton-proton (or $p-p$ ) process, Hans Bethe, a Jewish physicist who fled from Germany to United States in 1933, met and worked with George Gamow. Known for his ability to work out nuclear reactions on paper, Bethe made a name for himself publishing papers on nuclear processes. His expertise was soon recognized, leading to his appointment in 1939 to the theory division at Los Alamos, New Mexico, in charge of developing a nuclear bomb. Following the development of the atomic (fission) bomb, tested in 1945, attention turned to fusion, the result being the Hydrogen Bomb, successfully tested in 1948.

The H-Bomb proved that the proton-proton process worked, and the continuous radial heat of the Sun verified it. This triggered a new era in physics: if hydrogen could be fused to helium, perhaps helium nuclei could be fused to higher elements. An obvious process would lead to carbon $(\mathrm{C})$ :

${ }^{4} \mathrm{He}+{ }^{4} \mathrm{He}+{ }^{4} \mathrm{H}$ might yield ${ }^{12} \mathrm{C}$

The fusion of ${ }^{12} \mathrm{C}$ from three ${ }^{4} \mathrm{He}$ nuclei looked simple on paper, though the probability of a simultaneous collision and fusion of three nuclei seemed impossibly low. But a two-step process through Beryllium (Be) looked promising:

${ }^{4} \mathrm{He}+{ }^{4} \mathrm{He}$ would yield ${ }^{8} \mathrm{Be}+$ a gamma ray

${ }^{8} \mathrm{Be}+{ }^{4} \mathrm{He}$ would yield ${ }^{12} \mathrm{C}+$ a gamma ray

Once a pathway to ${ }^{12} \mathrm{C}$ had been developed, a more complex 6-step "carbon-carbon" $(c-c)$ process proposed by Bethe would yield Nitrogen (N) and Oxygen $(\mathrm{O})$ and multiple pathways to other elements. This series of ad hoc processes pointed the way to a comprehensive methodology while incidentally generating the four primary elements of life- $-\mathrm{H}, \mathrm{C}$, $\mathrm{N}$, and $\mathrm{O}$.

In the 1950s, a remarkably talented team came together: husband-and-wife astronomers Geoffrey and Margaret Burbidge, the experimental physicist William Fowler, and the British theoretical physicist Fred Hoyle, well known for his off-handed invention of the term "big bang." Over several months, they worked out a multitude of nuclear processes that might account for the fusion of elements in the Periodic Table. The result was a landmark 103-page paper published in The Physical Review (1957). ${ }^{17}$ Six decades later, the paper is so famous among physicists that it is known from its authors as B-squared-FH $\left(\mathrm{B}^{2} \mathrm{FH}\right)$. While they attempted to work out processes that would create all elements in the entire Periodic Table, they recognized a barrier at iron 26. Fusion of the lower elements resulted in energy emission; 
elements above iron 26 required an infusion of energy. They could guess at steps for heavy-element fusion but could only speculate about the precise source of the needed energy.

\section{The Hydrogen-Helium Mystery and the Big Bang}

Up until the $\mathrm{B}^{2} \mathrm{FH}$ paper, a long-term presence of foundational hydrogen was assumed, especially among astronomers such as Fred Hoyle who held steadfastly to the idea of an eternal universe. But George Gamow, Carl-Friedrich von Weizsacker, and Charles Critchfield - a generation of physicists of remarkable skill and intuition - saw things differently. Hydrogen provided a foundation for stellar fusion, but its origin was a mystery, emphasized by massive amounts available for star making. Related to this was the inordinate amount of helium across the universe which far exceeded what was deemed possible from stellar nucleosynthesis. In 1964 Fred Hoyle and Roger Taylor noted that synthesis of that much helium in stars would have led to galaxy brightness far in excess of what was observed. Their paper on the "mystery of cosmic helium abundance" asserted that "helium production in ordinary stars is inadequate to explain" the helium abundance observed. To assume hydrogen and helium as unexamined givens was too simple and lacked an empirical foundation. The conviction grew that there must be another process-indeed, another site-where hydrogen and helium in massive quantities came into existence. Hoyle and Taylor suggested a "radiation origin" for these foundational elements. ${ }^{18}$

We now know that the "radiation-origin" was the big bang, but from the 1940s until 1960s, the big bang remained a theory that took a back seat to the more widely held steady-state cosmology. In 1948 the theory of the big bang along with the mathematics of big-bang nucleosynthesis took shape at the hands of George Gamow and his former student Ralph Alpher. ${ }^{19}$ Later in the year, Alpher and Robert Herman published a brief note in Nature that included a general prediction of a remnant cosmic microwave background (CMB), including an estimate of its temperature at $5 \mathrm{~K}$ (five degrees above zero on the Kelvin scale), remarkably close to the present measurement of $3 \mathrm{~K} .{ }^{20}$ Alpher and Herman continued to explore the idea of a big bang, but no one else took particular note, and Fred Hoyle, who had derisively coined the term, stubbornly resisted the idea; thus it remained controversial until 1965 when Arno Penzias and Robert Wilson accidently discovered the CMB as a puzzling signal from deep space. ${ }^{21}$ When it finally took hold, it made its debut in 1967 as a "primeval fireball" in Scientific American. ${ }^{22}$ Once serious consideration and mathematical reconstruction of the big bang began later in the 1970s, the pure energy that necessarily prevailed at the moment it all began (time zero) became the focus of attention. Einstein's equivalency of energy and matter in his celebrated $\mathrm{E}$ $=\mathrm{mc}^{2}$ equation meant that hydrogen had precipitated from a precursor, an incredibly dense and hot energy field that underwent rapid expansion and density decline. As the theory developed, it seemed clear from the known laws of physics and the effects of falling heat and density that energy would precipitate as various particles, including hydrogen nuclei, and following intermediate production of deuterium and tritium, helium nuclei would fuse as long as temperature remained high enough. The result was an expanding cloud of debris consisting almost entirely of hydrogen and helium. ${ }^{23}$ Simple application of physical laws indicated that this was a temporary endpoint; fusion of helium into higher elements required temperatures in excess of 100 million degrees that were no longer available as big-bang material continued to expand.

Inordinate amounts of hydrogen and helium as the primary material of early stars now made sense, and still do: excluding the still-elusive dark matter, these two make up $99 \%$ of all ordinary matter even today. ${ }^{24}$ Further fusion in the stars and processes that burned (or cooked, baked, or broiled) the first twentysix elements had already been worked out, but in the real world of the early universe, sites for element cooking would not be available for several hundred 
million years and would depend on the formation of the earliest stars.

Building on a foundation of hydrogen and helium, the earliest stars took over the narrative. Star centers provide an environment where the pressure of gravity sends temperature soaring beyond the crucial 100-million-degree mark, allowing the cooking of helium to higher and heavier elements that will in turn sink to the center of the star. There, further fusion drives fusion up the Periodic Table. The $p-p$ and $c-c$ processes already described were then understood as steps in the evolution of stars in which they gradually developed an onion-like structure with the heaviest elements undergoing fusion at the core. Thus understood, the evolution of the elements was virtually inseparable from the life history of stars. By the end of the 1970s, fusion had settled into a twochapter narrative; the creation of $\mathrm{H}$ and He during the big bang; and the fusion of beryllium 3 to iron 26 at the centers of very large stars. This was clear. What was not clear was how nucleosynthesis could bypass the iron 26 barrier. Where would the needed energy come from?

The answer was tentatively proposed as early as the 1950s: a site so catastrophic that unimaginable energy would be available. Physicists nursed an intuition that stellar explosions might be the place, especially since the spectra from remnants of such explosions indicated the presence of heavier elements. But decisive proof was missing: heavier elements could have been present before the source star exploded. Until stellar explosions could be observed in real time, a possible third chapter in element fusion was theory not yet established as fact.

\section{Supernova Fusion}

Supernova explosions are a relatively rare event in the Milky Way. Speculation has identified the star that allegedly foretold the assassination of Julius Caesar (44 BCE) as a supernova, but celestial signs accompanying human events are common in mythology from Europe to India and China. In the context of science, multiple sightings are needed for verification. Until the late 20 th century three verifiable Milky Way supernovas were known by their remnants. The 1054 explosion that created the Crab Nebula, was described by the Chinese as a "guest-star"; it was seen also by the Babylonians, and, as a petroglyph at Chaco Canyon suggests, possibly by Native Americans. ${ }^{25}$ When Tycho Brahe's saw a supernova in 1572, he said he was "amazed . . . astonished and stupefied." Kepler observed one visible for a year in 1604; known as "Kepler's star" it was the most recent one until the twentieth century. For all of these, observations were limited to a light source so intense that it was visible even during daylight hours for several weeks or months, but these occurred too far in the past for reliable analysis. Waiting for one was discouraging because the Milky Way average seemed to run about one supernova every 250 years. From the limited timespan of human observation, this is a very long time, though there was little doubt about supernovas on a cosmic scale. Assuming that the Milky Way average applies to the estimated 350 billion large galaxies, the universe experiences 300 supernovas every second, possibly more: the Pinwheel Galaxy 20 million light years away has experienced an astonishing frequency of three supernovas since 1994, and 7 trillion dwarf galaxies would add to any estimate, raising it closer to Marcus Choun's suggested total of 1000 supernovas every second. ${ }^{26}$ Their importance in the overall narrative of cosmic history cannot be ignored.

Fortuitously, a supernova that could be studied with modern astronomical methods was observed in 1987 in a nearby dwarf galaxy, the Large Magellanic Cloud, at a distance of 168,000 light years. Prior to this event, no one was quite sure about the demise of stars. In 1961, Alan Broms admitted that "what brings it [the end of a star] about we do not know, though we do assume it is some condition which upsets the balanced state of the star's interior." 27 By the late twentieth century the physics of core collapse had been worked out with some variation dependent on the size of the star. ${ }^{28}$ Stars in the size range of the Sun last billions of 
years, slowly fusing hydrogen to helium, and perhaps a few of the lower elements. But in larger stars of nine or more solar masses, fusion continues until an energy barrier at iron 26 causes a material pileup that increases until fuel runs out. As long as fusion continues, the production of energy at the stellar core provides upward pressure against the downward force of gravity, but once fusion stops, gravity takes over. The atomic structure of the iron core cannot withstand the pressure; the electron shells break up; the vast interior space of the atoms is eliminated, and a catastrophic gravitational collapse ensues, with inward velocity approaching 70,000 kilometers (45,000 miles) per second. In a microsecond, a stellar core the size of the earth is reduced to the size of Manhattan or Singapore. This collapse is so violent, with energy and heat produced at such enormous levels, that a rebound and supernova explosion follows, with debris ejected into space at an estimated ten percent the speed of light approximately $30,000 \mathrm{~km}$ (18,000 miles) per second.

Prior to the 1987 supernova, precisely what might happen during the demise of a star was little more than speculation. Broms admitted that "we are doing much guessing about these last stages and final endings of the star careers," but he made an intuitive guess: "It may well be that within them the heavy elements are formed by higher temperature transmutations." 29 Writing about the same time, Norman Berrill was more committal: with the explosion of a red giant star as a supernova, it "adds to the space outside, so that stardust now consists of the whole range of the elements in roughly the proportions we are familiar with on earth." 30 By 1983 Nigel Calder could say quite confidently, "more massive stars [than the sun] come to a dramatic end in a supernova explosion that manufactures heavy elements and flings many of them out into space." ${ }^{31}$ But qualifications appeared in the late $20^{\text {th }}$ century that limited supernova fusion to elements from hydrogen 1 to zinc $30 .^{32}$ The problem arose from the enormous velocity of supernova expansion; as John and Mary Gribbon point out, "the normal pattern of light and dark lines in the spectrum get smeared out by huge Doppler shifts into broad bands of light and dark." 33 Precise identification of specific heavy elements is thus problematic. In 2003, a Carnegie Symposium on the Origin and Evolution of the Elements was attended by more than 130 scientists. Every phase of the process was examined; a collection of papers was produced by Cambridge University Press. Notably, the production location of the heaviest elements could not yet be determined; as Bernard Pagel confirmed in the conference summary, the r-process is basic to heavy element fusion, but he acknowledged that "many mysteries" remain, adding that "there is no certainty as to where it occurs (italics added)." ${ }^{34}$

Despite uncertainty among astrophysicists, the much-repeated popular view that every element above iron has a supernova origin has prevailed. This idea has been passed on like a baton for half a century; the list of well-known scientists and cosmologists who repeated it is rather astonishing: Preston Cloud, Carl Sagan, Timothy Ferris, Alan Guth, Neil de Grass Tyson, Eric Chaisson, and John Hands. ${ }^{35}$ Big History presentations, including the McGraw Hill textbook, repeat this view. ${ }^{36}$ As recently as the May 2, 2017 , publication of Quarks to Culture, Tyler Volk could write, "Upon explosion, supernovas disperse their created products outward. The result is approximately eighty types of stable nuclei, a number based on how many protons each contains." ${ }^{37}$ The long life of this tenuous assumption is easy to explain: no additional site for fusion was known; the universe contained all 92 elements; therefore, supernovas must be the site where all the heavy elements were born. The assumption thus became conventional wisdom that brought to completion a three-part narrative for the creation of the elements: (1) hydrogen 1 and helium 2 creation during the big bang; (2) lithium 3 to iron 26 fusion at the centers of large stars; and (3) nickel 27 to uranium 92 during supernova explosions.

\section{Neutron Star Merger and Element Fusion}

And so the story stood, but the science story 
is usually tentative, rarely complete. Writing in 2010, B. D. Metzger et al anticipated gravity waves from a collision of neutron stars, along with an electromagnetic display from heavy-element fusion. ${ }^{38}$ When it happened on August 17, 2017, a fourth and corrective chapter was written and the dramatic term "kilonova" moved to center stage - the name referring to luminescence a thousand (kilo) or more times greater than any other cosmic event. Gamma-ray bursts that had been detected over several decades showed that black-hole creation and black-hole-neutron-star (BHNS) collisions may have occurred, but the Kilonova 2017 event was a NS-NS collision, the first observed in real time. As objects too small to be observed even through the most powerful telescope, neutron stars are known from rapid light pulses flashed across the universe-from which they derive their name as "pulsars." As a neutron star rotates, pulses are sent out like lighthouse beams visible if sent in the direction of the earth; their frequency - up to several hundred times per second-tells us that neutron stars have preserved the angular momentum of their much larger originals and thus spin at unimaginable velocities. ${ }^{39}$

Neutron stars are downtown-city-size supernova cinders. Typically 8 to 12 miles in diameter, they contain protons and electrons compressed so densely into neutrons that all interior space has been eliminated; they are so weighty and solid that a teaspoon full would weigh up to a billion tons, a potful would outweigh a mountain —within Earth's gravitational system. Their gravitational force on any common object would result in distortion and stretching, or what Nigel Calder once called "spaghettification." 40 Their density dictates masses akin to sun-like stars: estimates have put the two observed on August 17 at 1.6 and 1.1 times the mass of the Sun. They were nothing less than cosmic bombs waiting for ignition.

Given the frequency of supernova explosions over 10 to 12 billion years, millions of neutron-stars may be wandering through the Milky Way, every other galaxy, and possibly intergalactic space. Survival of astronomical objects for $10+$ billion years is not unusual; the space between stars is so greatthousands or millions of times their diameters - that stellar collisions are rare, even when galaxies merge. Statistically, even close encounters of neutron stars are rare. Analysis indicates the two detected almost certainly originated in the same galaxy, NGC 4993. ${ }^{41}$ Precisely how two of these leftover cinders from widely separate events found each other is beyond discovery-the odds are miniscule-but once together, they formed a binary system that may have spun for millions of years until orbital decay doomed them to catastrophic collision. Estimates place neutron star mergers at several magnitudes fewer than supernovas, but the vastness of the universe assures regular occurrences.

The detection of gravity waves increasing from 24 Hertz to several hundred over just 100 seconds provided a first-time record of inspiraling neutron stars in a tightening orbit at breakneck speed, dozens of rotations per second. The 1.7 second silence marked the final death plunge and collision at close to the speed of light signaled by the two-second gammaray burst. Astrophysical theory suggests that the bulk of material from merging neutron stars totaling more than three solar masses will disappear into a black hole; meanwhile a starburst of radiation equal to billions of stars signaled an explosion of material blasted into surrounding space at 20 to $30 \%$ the speed of light $-40,000$ to 50,000 miles per second. Spectral analysis indicated rapid nucleosynthesis that peaked during the first day. ${ }^{42}$ Fusion may have retraced fundamental pathways (the $p-p$ and $c-c$ processes, etc.) with new pathways made possible by kilonova conditions.

Colliding neutron stars provide a unique site for fusion different from supernovas - a neutron-rich environment for building higher elements with heat the engine driving particle collisions. As neutrons are propelled into the nuclei of unstable isotopes, nuclear decay provides a countervailing force: dozens of isotopes have half-lives of microseconds, making sustained nuclear construction tenuous. But the rapid 
Artist depiction of neutron-star collision. Note gravitational waves on the background matrix, laser-like pulsar beams, and gold and heavy element ejecta. Acknowledgements: National Science Foundation (NSF) / Laser Interferometer Gravitational Wave Observatory (LIGO) Sonoma State University / A. Simonnet.

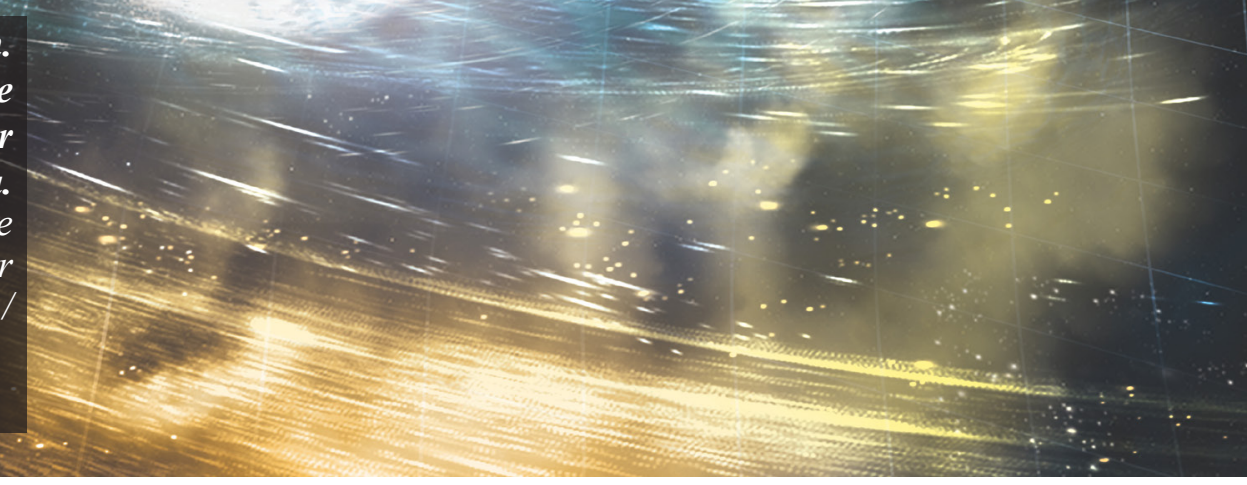

neutron process ( $r$-process) explored in detail in $\mathrm{B}^{2} \mathrm{FH}$ bypasses this difficulty. Interestingly, the $\mathrm{B}^{2} \mathrm{FH}$ team recognized the need for "a large flux of neutrons ... available in a short period of time," adding that "the precise source of the neutrons is not important. . . . any source capable of supplying a large neutron flux on a short time-scale of order 10-100 sec, would meet the requirements." 43 Sixty years before any idea of a kilonova existed and the first one was observed, the $\mathrm{B}^{2} \mathrm{FH}$ theorists defined the precise environment 
needed for heavy-element nucleosynthesis.

Forced tunneling of neutrons into nuclei faster than they can decay builds isotopes by the hundreds while beta decay turns some neutrons into protons. The resulting presence of both neutrons and protons allows for conversion of the nucleus to the next element on the Periodic Table. An unremitting barrage of neutrons thus provides a foundation for a sequential fusion of stable nuclei that effectively carve a pathway through radioactive decay, leading to the fusion of roughly half the heavy elements on the Periodic Table. ${ }^{44}$ Spectral analysis shows an estimated 16,000 earth masses of higher elements created, including the lanthanide sequence of rare-earth elements, with gold and platinum estimated at four earth masses - a mineral display of unfathomable wealth. ${ }^{45}$ As we look at treasured necklaces and rings of gold, we are looking at the fabulous riches of ancient kilonovas.

\section{Conclusion}

In retrospect, the first step of hydrogenhelium creation was missing from Fred Hoyle's 1946 paper: he thought the big bang was a mistake and never accepted it, even after the Cosmic Background Explorer (COBE) sent back an image of the Cosmic Microwave Background (CMB). His paper was heavily mathematical and focused on hydrogenhelium fusion in what he called "the helium zone." But he was essentially right in recognizing stars as places where element evolution occurs. A brilliant generation of physicists continued his work, recognizing that the conditions required for constructing higher and heavier elements varied enormously — so much so that a single site for element building was impossible. The task of sorting through the possibilities has taken three quarters of a century and eventually depended on the construction of more powerful instruments that could bring observations of distant events down to earth. Our earliest observations through the Mount Wilson and Palomar telescopes were limited to the narrow range of visible light; the gravitational waves from Kilonova 2017 perturbed the space-time framework where we reside one-thousandth of the width of a proton. We have indeed come a long way since the Greek story of a fiery chariot driven across the sky by the sun-god Helios and the belief that stars were lights flickering from a celestial dome. Now we know that the fusion of the whole range of elements has required four distinctively different environments, all of them occurring in unearthly places during events that are truly catastrophic. The kilonova chapter of the element-building story appears to complete the other three, though there remain puzzles and pathways not entirely sorted out. New atom smashers will increase understanding of heavy-element nucleosynthesis. One day we may have to write another chapter.

For those engaged in Big History, this merger of neutron stars and the resulting kilonova have completed the story of nucleosynthesis. The generation of the elements has profound importance for subsequent history, but this is easily passed over as if unimportant. Thus we read in a brief history of the universe by a prominent astrophysicist that "rapidly evolving massive stars are responsible for producing most of the heavy elements, such as carbon, oxygen, and silicon, from which we are made."46 The added italics highlight the compression and virtual loss of the larger narrative that adds significance to the element creation story. It seems endemic to the sciences to confine observation to disciplinary silos, thus losing the most relevant connections that justify the investigation of cosmic history and provide meaning for discoveries. It is the role and task of Big History to bridge these academic silos - to see, understand, and communicate the unifying narrative that makes sense of the fragmentary knowledge of discipline-defined knowledge.

From ninety-two elements, a virtually infinite range of chemical molecules have been built - in space, and more profusely in the congenial environments of Earth. Somewhere around four billion years ago, clusters of molecules, activated by continuous energy flows, acquired identity and pattern retention sufficient to maintain themselves and reproduce, and 
the nearly interminable era of single-celled life was set in motion. Cells, we now know, are constructed from up to 100 trillion atoms, and when linked up to form living organisms, we must multiply by hundreds of trillions more. Ultimately, though, such life, from trilobites to tarantulas, plankton to primates, is built on the foundation of the elements - life's emergent skills, motivations, thoughts, and imaginings firmly nested within the mysterious properties of the elements themselves. And while the elements basic to life are clustered in the lower twenty six, our creative energies have found uses for virtually all the others. What would life be without silver, gold, and platinum? Kilonova 2017 has indeed moved us a giant step closer to what Thomas Berry called the New Story-a captivating narrative of cosmic history. ${ }^{47}$

\section{Endnotes}

1. B. P. Abbott et al., "GW170817: Observation of Gravitational Waves from a Binary Neutron Star Inspiral," Physical Review Letters, 119 (16 November 2017), 1-18.

2. D. A. Coulter, et al., "Swope Supernova Survey 2017a (SSS17a), the Optical Counterpart to a Gravitational Wave Source," Science 358, Issue 6370 (22 December 2017), 15561558.

3. Eva Botkin-Kowacki, "Neutron Star Discovery Marks Breakthrough for "Multi-messenger Astronomy," The Christian Science Monitor (16 October 2017).

4. Li-Xin Li, Bohdan Paczynski, "Transient Events from Neutron Star Mergers," The Astrophysical Journal Letters Volume 507 (1 September, 1998).

5. Adrian Cho, "Trio Surfs Gravitational Waves to Nobel Glory," Science 355, issue 6359 (6 October 2017), 17.

6. Metzger, B. D.; Martínez-Pinedo, G.; Darbha, S.; Quataert, E.; et al. (August 2010). "Electromagnetic counterparts of compact object mergers powered by the radioactive decay of r-process nuclei". Monthly Notices of the Royal Astronomical Society. 406 (4): 2650-2662.

7. B. P. Abbott $[+4000$ coauthors $]$, "Multi-messenger Observations of a Binary Neutron Star Merger," The Astrophysical Journal Letters 848, Number 2 (16 October 2017), 1-147.

8. Adrian Cho, "2017 Breakthrough of the Year: Cosmic Convergence.” Science 358, Issue 6370 (22 December 2017), 1520-1521.

9. Darrell D. Ebbing, Steven D. Gammon, General Chemistry, $9^{\text {th }}$ edition (New York: Houghton Mifflin, 2009), 857.

10. Barry Wood, "Bridging the 'Two Cultures': The Humanities, Sciences, and the Grand Narrative." International Journal of Humanities Education, Vol. 10 (2013), 44-55.

11. Fred Hoyle, "The Synthesis of the Elements from Hydrogen." Monthly Notices of the Royal Astronomical Society, Vol. 106 (1946), 343-369.

12. Margaret Burbidge, "Synthesis of the Elements in Stars: $\mathrm{B}^{2} \mathrm{FH}$ and Beyond," Origin and Evolution of the Elements, ed. Andrew McWilliam (UK: Cambridge University Press, 2004), 8 .

13. Hoyle, 353. 
14. Andrew Robert Steere, A Timeline of Major Particle Accelerators. Master of Science Thesis (Michigan State University, 2005).

15. An element's atomic number refers to its traditional position (1 to 92) on the Periodic Table. The use of preceding superscripts which follows astrophysical practice refers to the total protons + neutrons in a stable nucleus; with the sole exception of hydrogen, the nuclear total is always double the atomic number. Thus carbon 6 is No. 6 on the Periodic Table while its nuclear total is coded ${ }^{12} \mathrm{C}$. This symbolism is useful for designating isotopes; thus the radioactive form of carbon, ${ }^{14} \mathrm{C}$, is a well-known isotope that contains two extra neutrons in the nucleus.

16. Amos Harpaz, Stellar Evolution (Wellesley, MA: A. K. Peter, 1994), 48-50.

17. Geoffrey Burbidge, Margaret Burbidge, William Fowler, Fred Hoyle, "Synthesis of Elements in Stars" [B'FH], Review of Modern Physics, Vol. 29 (1957), 547-650.

18. Fred Hoyle and Roger J. Taylor, "The Mystery of the Cosmic Helium Abundance," Nature 203 (12 September 1964), 11081110 .

19. R. A. Alpher, H. Bethe, George Gamow, "The Origin of Chemical Elements." Physical Review Vol. 73, No. 7 (April 1, 1948), 803-804. Gamow and Alpher wrote the article, but Gamow, a prankster, raised the article to fame by adding Hans Bethe to create an authorial trilogy (Alpha-Bethe-Gamow), a pun on the first three letters of the Greek alphabet $(\alpha \beta \gamma)$. Its publication on April Fools Day enhanced the joke.

20. R. A. Alpher, R. Herman "Evolution of the Universe," Nature 162 (November 13, 1948), 774-775.

21. Aldo Penzias, Robert Wilson, "A Measurement of Excess Antenna Temperature at $4080 \mathrm{Mc} / \mathrm{s}$," Astrophysical Journal 142 (1965), 419-421. For a summary of pre-1965 reticence about the term big bang, see Barry Wood, "Imagining the Unimaginable: Narratives of the Big Bang," Big History Journal, Vol. 2, No. 1 (Spring 2017), 1-13.

22. P. J. E. Peebles and David T. Wilkinson, "The Primeval Fireball," Scientific American 216 (June 1967), 28-38.

23. We now know that big bang material contained trace amounts of lithium 3 but this had negligible effect on subsequent fusion.

24. Steven Weinberg, The First Three Minutes, updated edition (New York: Basic Books, 1993), provides a definitive account of $\mathrm{H}$ and He creation during the big bang; see also Joseph Silk, The Big Bang. $3^{\text {rd }}$ edition (W. H. Freeman, 2001).
25. John C. Brandt \& Ray A. Williamson, The 1054 Supernova and Native American Rock Art, Astroaeoastronomy, No. 1, Journal for the History of Astronomy, Supplement, 10 (1979), $1-38$.

26. Marcus Chown, The Magic Furnace (New York: Oxford University Press, 2001), 203.

27. Allan Broms, The Emerging Universe (New York: Doubleday, 1961), 178.

28. Heger, Alexander, et al., "How Massive Single Stars End Their Life”. Astrophysical Journal. 591 (2003): 288.

29. Broms, 80, 83.

30. Norman Berrill, You and the Universe (New York: Holt, 1958), 178.

31. Nigel Calder, Timescale: An Atlas of the Fourth Dimension (New York: Viking, 1983), 279.

32. Timmes, F. X.; Woosley, S. E.; Weaver, Thomas A. (1995). "Galactic Chemical Evolution: Hydrogen through Zinc". Astrophysical Journal Supplement Series. 98 (1995), 617.

33. John and Mary Gribbon, Stardust: Supernovae and Life-the Cosmic Connection (New Haven: Yale: 2000), 164.

34. Bernard E. J. Pagel, "Conference Summary," Origin and Evolution of the Elements, 483.

35. Preston Cloud, Cosmos, Earth and Man (New Haven: Yale University Press, 1978), 11, 26; Carl Sagan, Cosmos (New York: Random, 1980), 233; Timothy Ferris, Coming of Age in the Milky Way (New York: William Morris, 1988), 279; Alan H. Guth, The Inflationary Universe: The Quest for a New Theory of Cosmic Origins (Reading, MA: Helix Books, 1997), 98-99; Neil deGrasse Tyson and Donald Goldsmith, Origins: Fourteen Billion Years of Cosmic Evolution (New York: Norton, 2004), 165; Eric Chaisson, Epic of Evolution (New York: Columbia University Press, 2006), 171; John Hands, Cosmosapiens: Human Evolution and the Origin of the Universe (New York: Overlook Duckworth, 2015), 131.

36. David Christian, a Cynthia Stokes Brown, Craig Benjamin, Big History: Between Nothing and Everything (New York: McGraw Hill, 2014), 28.

37. Tyler Volk, Quarks to Culture: How We Came to Be (New York: Columbia University Press, 2017), 46.

38. B. D. Metzger et al, "Electromagnetic Counterparts of Compact Object Mergers Powered by the Radioactive Decay of r-process Nuclei," Monthly Notices of the Royal Astronomical Society (27 April 2010), 1-15. 
39. The highest neutron-star rotation so far observed is 716 times per second.

40. Nigel Calder, The Key to the Universe: A Report on the New Physics (New York: Viking, 1977), 199.

41. B. P. Abbott, et al., "On the Progenitor of Binary Neutron Star Merger GW 170817," The Astrophysical Journal Letters, Vol. 850: Letter 40 (1 December 2017), 1-18.

42. Iair Arcavi et al, "Optical Emission from a Kilonova Following a Gravitational-wave-detected Neutron-star Merger," Nature 551 (16 October 2017), 1-24.

43. $\mathrm{B}^{2} \mathrm{FH}, 587$.

44. Daniel Kasen et al, "Origin of the Heavy Elements in Binary Neutron-star Mergers from as Gravitational-wave Event," Nature 551 (2 November 2017), 80-84.

45. Robert Sanders, “Astronomers Strike Cosmic Gold," Berkeley News (16 October 2017), 1-10; Adrian Cho, "An Earthly Search for Gold's Cosmic Origins," Science, Vol. 358, Issue 6366 (24 November 2017), 981-982.

46. Joseph Silk, A Short History of the Universe (New York: Scientific American Library, 1994), 217-218.

47. Thomas Berry, "The New Story," The Dream of the Universe (San Francisco: Sierra Club Books, 1988), 123-137. 
